\title{
环戊二烯衍生物的合成及应用研究进展
}

\author{
叶俊伟黄雪明王萧潇郑婷 \\ 高远 贡卫涛宁桂玲*
}

(大连理工大学化工与环境生命学部化工学院 精细化工国家重点实验室 大连 116024)

\begin{abstract}
摘要 环戊二烯及其衍生物是一类重要的小分子环烯烃化合物，在金属茂化合物合成、有机中间体合成和有机光电材 料等领域具有重要应用价值. 结合本课题组的研究成果, 主要综述环戌二烯衍生物的合成研究进展, 并介绍一些典型 的环戌二烯衍生物在有机合成领域应用研究进展.
\end{abstract}

关键词 环戊二烯衍生物；合成；应用；金属茂化合物；有机中间体

\section{Research Progress on the Synthesis and Application of Cyclopentadiene Derivatives}

\author{
Ye, Junwei Huang, Xueming Wang, Xiaoxiao Zheng, Ting \\ Gao, Yuan Gong, Weitao Ning, Guiling*
}

(State Key Laboratory of Fine Chemicals and School of Chemical Engineering, Faculty of Chemical, Environmental and Biological Science and Technology, Dalian University of Technology, Dalian 116024)

\begin{abstract}
Cyclopentadiene and its derivatives are a type of important small molecule cyclic olefin compounds, which have been widely applied in many fields such as synthesis of metallocene compounds, synthesis of organic intermediates and organic photoelectric materials. Based on our recent research results, in this review, the research progress on the synthesis of cyclopentadiene derivatives and the application of some typical cyclopentadiene derivatives in the field of organic synthesis are summarized.
\end{abstract}

Keywords cyclopentadiene derivatives; synthesis; application; metallocene compounds; organic intermediates

环戊二烯及其衍生物由于具有独特的五元环与共 轭双键结构, 使其既没有三元环和四元环化合物的高反 应活性, 也没有芳香性六元苯环的稳定性, 其化学稳定 性介于二者之间, 成为一类重要的有机合成反应原料和 中间体. 环戊二烯及其衍生物可以进行聚合 ${ }^{[1]}$ 、氢化还

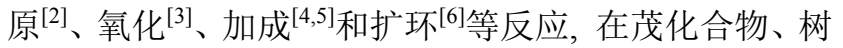
脂、农药、橡胶、阻燃剂、医药和光电器件领域等领域 已得到广泛应用 ${ }^{[7 \sim 9]}$.

单环戊二烯分子性质活泼, 在常温下即可聚合成二 聚环戊二烯(双环戊二烯), 超过 $100{ }^{\circ} \mathrm{C}$ 时将进一步聚合 生成三聚体、四聚体等. 工业中常将来源于煤炼焦过程 所得粗苯中的苯前馏分和石油裂解中 C5 馏分的双环戊
二烯粗产品为原料制备较高纯度环戊二烯. 首先将粗产 品通过蒸馏除去高组分, 再经热聚和解聚法得到高纯度 环戊二烯. 目前生产高纯度环戊二烯仍存在面临二聚耗 时长、分离、储存难等问题. 这极大地限制了环戊二烯 作为有机合成中间体和有机功能分子的应用，因此开发 新的环戊二烯及其衍生物的合成路线研究一直吸引着 人们极大的关注 ${ }^{[10 \sim 13]}$.

近几年，我们课题组设计合成了系列多芳基取代环

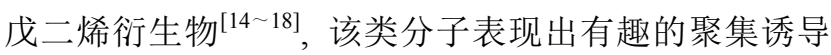
发光增强性质，在有机电致发光领域具有很好应用价 值 ${ }^{[17]} ;$ 同时该类分子可以通过酸或者 $\mathrm{Fe}^{3+}$ 催化氧化一

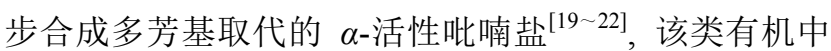

\footnotetext{
*E-mail: junweiye@dlut.edu.cn; ninggl@dlut.edu.cn

Received December 9, 2015; revised January 22, 2016; published online February 2, 2016.

Project supported by the National Natural Science Foundation of China (Nos. 51003009, 20772014), and the Fundamental Research Funds for the Central Universities of China (No. DUT14LK32) and the Science and Technology Research Foundation of Education Department of Liaoning Province (No. L2014033).

国家自然科学基金(Nos. 51003009, 20772014)、中央高校基本科研业务费(No. DUT14LK32)和辽宁省教育厅科学技术研究(No. L2014033)资助项目.
} 
间体具有独特的反应活性 ${ }^{[23 ~ 28]}$. 结合最近几年研究成 果，本文依照合成反应过程中有无金属催化进行分类， 归纳了环戍二烯及其衍生物的合成研究进展, 合成方法 包括 1,3-环龙二酮法、香豆素与炔反应法、烯炔反应法、 环己醇缩环法、醛酮缩合法、二聚体解聚法和光诱导扩 环法等, 并对一些典型环戊二烯衍生物在有机合成领域 的应用作以介绍.

\section{1 金属催化环戊二烯及其衍生物的合成}

\section{1 香豆素与炔反应法}

香豆素衍生物具有良好的生物活性，具有抗病毒、 抗微生物和抗肿瘤等性能, 已被广泛用于香料、医药和 农药等领域. 2014 年, Peng 等 ${ }^{[29]}$ 在二甲基亚砜(DMSO) 溶剂中, $80{ }^{\circ} \mathrm{C}$ 和钯催化条件下将 4-羟基取代的香豆素 和二苯乙炔环化生成得到化合物 1 (Eq. 1). 钯催化具有 导向多样性, 能够合成多种功能性的分子 ${ }^{[30]}$, 这种方法 具有条件温和，原料易得等优点.<smiles>[R]c1cccc2c1O[C+](=O)C=C2O</smiles>

\subsection{1,3-环戊二酮法}

环戊二酮衍生物可在六甲基二硅基胺基钾 (KHMDS)中和 $N$-(5-氯-2-吡啶基)双(三氟甲烷磺酰亚 胺 $)\left(\mathrm{ClPyN}(\mathrm{Tf})_{2}\right)$ 反应, 再在三 (二亚苄基丙酮)二钯 $\left[\mathrm{Pd}_{2}(\mathrm{dba})_{3}\right]$ 和三环已基膦 $\left[\mathrm{P}(\mathrm{Cy})_{3}\right]$ 催化条件下与芳基硼 酸反应生成环戊二烯衍生物 ${ }^{[31]}$ (Scheme 1).

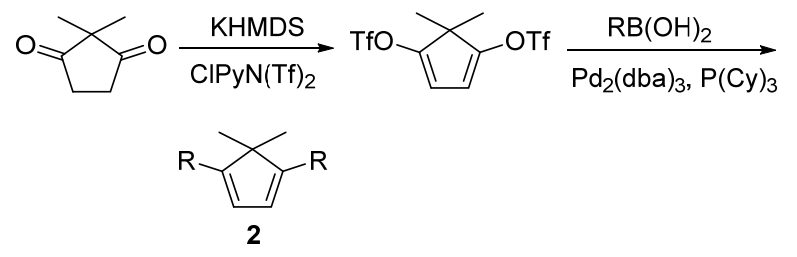

图式 1 1,3-环戊二酮法合成环戊二烯衍生物 Scheme 1 Synthesis of cyclopentadiene derivatives by 1,3-cyclopentadione reaction

\section{3 烯炔反应法}

均相的 $\mathrm{Au}(\mathrm{I})$ 和 $\mathrm{Au}(\mathrm{III})$ 催化剂在各种不对称反应中 显示出了很好的应用前景 ${ }^{[32]}$. 环戊二烯的取代类型影 响其反应活性 ${ }^{[33]}$, 手性取代基团会赋予环成二烯独特 的结构和性能. 2014 年, Wirtanen 等 ${ }^{[12]}$ 以三氟甲磺酸乙 烯酯和炔丙胺的衍生物为原料在 $\mathrm{Au}$ 催化条件下合成了 手性环戊二烯衍生物(Scheme 2). 从结构中可以看出手
性从炔丙基位置转移到环戊二烯环上.

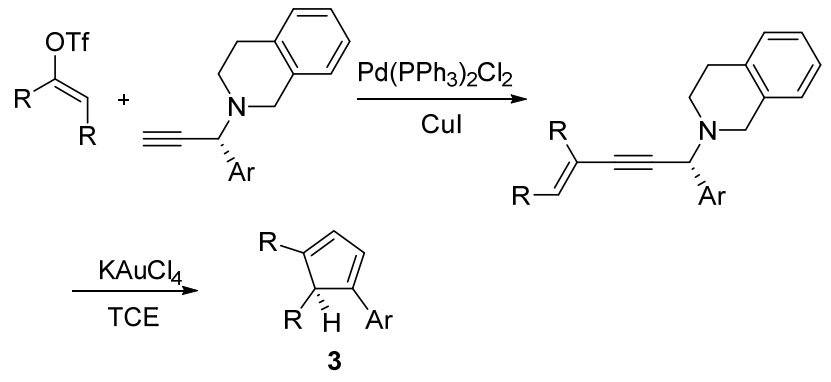

图式 2 烯炔反应法合成环戊二烯衍生物

Scheme 2 Synthesis of cyclopentadiene derivatives by olefin and alkyne derivatives reaction

\section{4 环己醇缩环法}

甲基环戌二烯具有优越的性能，广泛应用于燃料、 催化剂和医药等领域. Kryukov 等 ${ }^{[34]}$ 以环己醇为原料合 成了甲基环戊二烯. 在 $450{ }^{\circ} \mathrm{C}$ 条件下, 环已醇经氧化铝 催化可得甲基环戊烯, 然后经钼催化氧化再脱水得 5-甲 基环戊二烯. 反应式如 Scheme 3 所示.

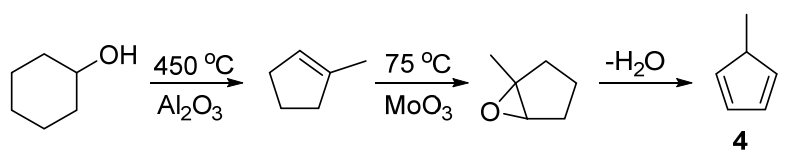

图式 3 5-甲基环戊二烯的合成

Scheme 3 Synthesis of 5-methyl-cyclopentadiene

\section{5 醛酮缩合法}

我们课题组利用醛酮缩合在 $\mathrm{Zn}$ 催化剂条件下合成 了多取代环戊二醇，再在酸性条件下合成了一系列多芳 基环戊二烯衍生物 ${ }^{[14 ~ 18]}$ (Scheme 4), 此方法具有原料廉 价、易得，产物稳定等优点. 此类化合物具有良好的聚 集诱导发光增强的性能 ${ }^{[14 \sim 16]}$, 可以用于有机电致发光 器件 ${ }^{[17]}$. 例如 1,2-二苯基-4-(4-甲氧基苯基)-1,3-环戊二 烯固体苂光量子效率相对溶液中的苂光量子效率成倍 的增加, 更有趣的是通过溶剂挥发法和再沉淀法可得到 不同形貌的发射蓝光的纳米晶 ${ }^{[18]}$, 在纳米材料领域展 现出较好的应用前景.

此外, 我们又利用 Suzuki 交叉偶联反应 ${ }^{[35]}$ 合成了 以下具有特殊官能团的多芳基环戍二烯(Eq. 2).

\section{2 非金属催化环戊二烯及其衍生物的合成}

\section{1 二聚体解聚法}

环戊二烯二聚体可发生加成反应合成重要的中间 产物或产品应用于工业生产，如生产涂料、不饱和树脂、 金刚烷、燃料等 ${ }^{[36]}$. 在加热条件下它可解聚生成单环戊 二烯. 工业中常采用裂解 C5 馏分获得环戊二烯, 但此 


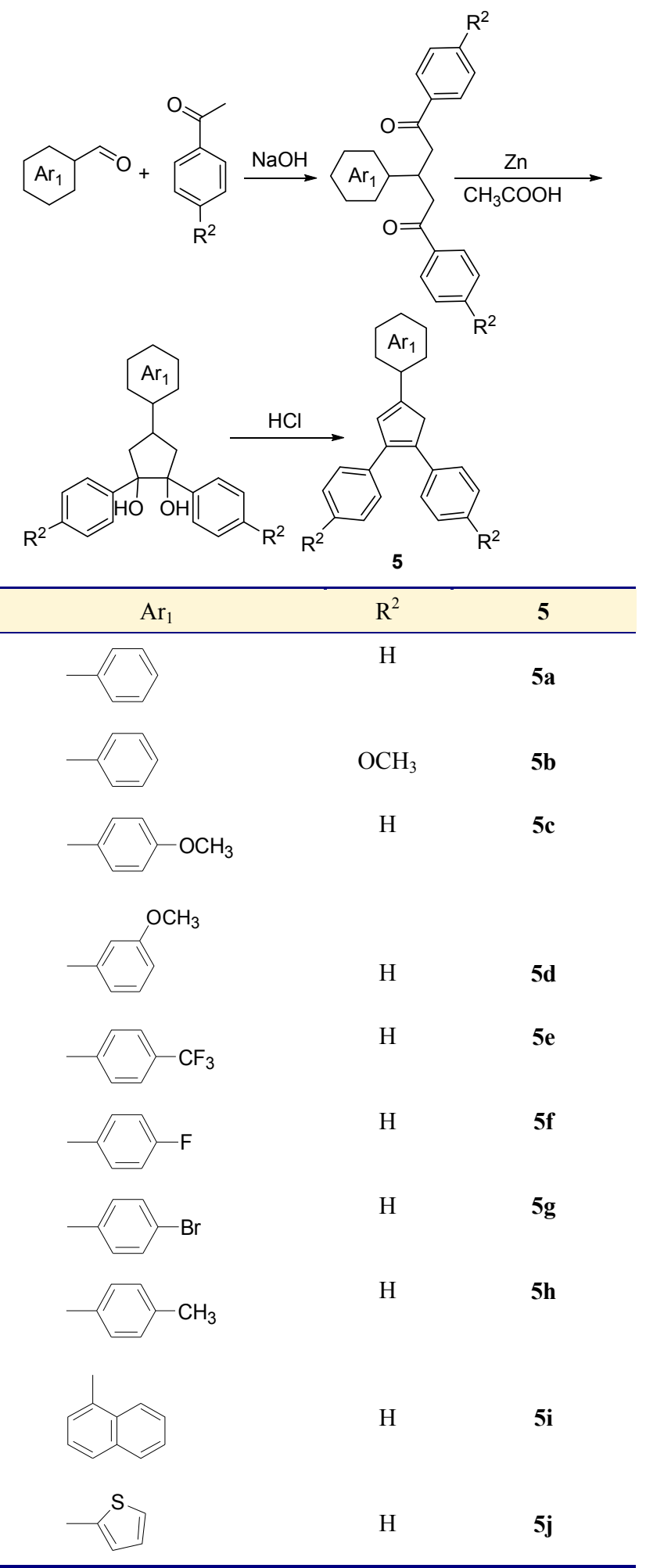

图式 4 醛酮缩合法合成环戊二烯衍生物

Scheme 4 Synthesis of cyclopentadiene derivatives by aldehydes and ketones condensation reaction

法所得环戊二烯不纯, 需将环戊二烯加热聚合, 再经解 聚获得纯化的环戊二烯.

由于环戊二烯和其二聚体具有重要的应用价值, 环

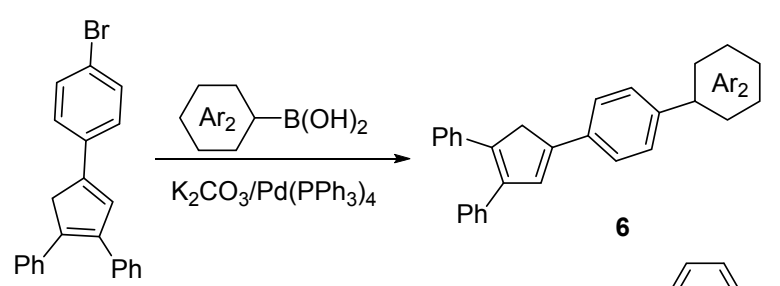<smiles>C1=C(c2ccc(-c3ccccc3)cc2)CC(c2ccccc2)=C1c1ccccc1</smiles>

$6 a$

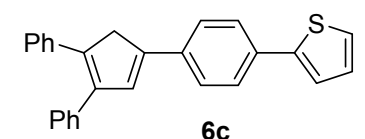

$6 c$<smiles>C1=C(c2ccccc2)CC(c2ccc(-c3cccc4ccccc34)cc2)=C1c1ccccc1</smiles>

$6 b$
戊二烯解聚的反应机理被广泛研究 ${ }^{[37]}$. Khambata 等 ${ }^{[38]}$ 研究了逆反应的动力学常数, 确定了反应向逆向发生的 温度范围，并提出了单分子反应途径. 2010 年, $\mathrm{Krupka}^{[39]}$ 研究了正向反应的动力学常数, 这为环戊二 烯在常温下易聚合为二聚体这一反应提供了理论依据. 同时，环戊二烯二聚体的解聚方法也不断被优化. 2013 年, Goswami 等 ${ }^{[40]}$ 报道了利用光化学反应控制二聚环戊 二烯转化为环戊二烯的反应(Eq. 3). 此方法具有条件易 控制、操作简单和节约能源等优点. 光化学诱导生成环 戊二烯的反应是一类新型且具有广阔应用前景的反应, 为低能耗、高效率的工业化生产奠定了良好的基础.

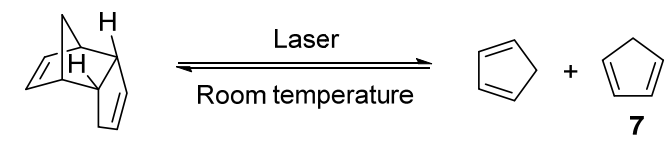

环戊二烯二聚体存在桥式和挂式二种同分异构体, 其中桥式环戍二烯二聚体更易解聚为环戊二烯 ${ }^{[41]}$. 2013 年, Wang 等 ${ }^{[42]}$ 通过将易得的桥式双环戊二烯直接氢化、 异构化制备得高闪点和低凝固点的高能燃料挂式-四氢 二聚环戊二烯. 此方法具有操作简单、高效率和低污染 等优点. 该类化合物有可能被应用于动力燃料. 2015 年, Wang 等 ${ }^{[43]}$ 指出可将两种不同形式的环戊二烯二聚体通 过与环戊二烯聚合得到不同结构的三聚环戊二烯，再经 过进一步还原、转化可得到挂式一四氢三聚环戊二烯. 这 种方法具耗时短, 易分离和溶剂用量少等优点, 具有潜 在的工业应用价值.

\section{2 光诱导扩环法}

Mendez 等 ${ }^{[44]}$ 用乙烯基三苯基环丙烯在光化学诱导 条件下环膨胀合成了 1,2,3-三苯基环成二烯, 结构式如 Eq. 4 所示. 
<smiles>C=CC1(c2ccccc2)C(c2ccccc2)C1c1ccccc1</smiles>

该反应在反应过程中释放了环丙烯张力, 并且乙烯 基三苯基环丙烯两个单独的双键在此过程中产生了共 轭双键, 使化合物的能量降低. 因此, 在光化学诱导条 件下，乙烯基三苯基环丙烯具有扩环生成三苯基环成二 烯的趋势.

\subsection{2,4-环己二烯酮法}

烯酮作为热力学稳定性好和动力学活性高的化合 物, 常用于电环化反应和亲核加成化学 ${ }^{[45]} .2012$ 年, Scheer 等 ${ }^{[46]}$ 将苯酚异构化合成了 2,4-环己二烯酮, 然后 在加热条件下脱一氧化碳得到环戊二烯(Scheme 5).

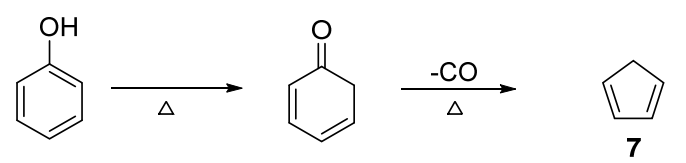

图式 5 苯酚合成 1,3-环戊二烯

Scheme 5 Synthesis of 1,3-cyclopentadiene from phenol

2014 年, Koch 等 ${ }^{[47]}$ 通过将 2-羟基-苯甲酸甲酯真空 热解(FVT)脱去一分子 $\mathrm{MeOH}$ 得 2,4-环己二烯酮, 然后 再经真空热解脱去 $\mathrm{CO}$, 再与甲醇反应最终得到化合物 9 (Scheme 6).

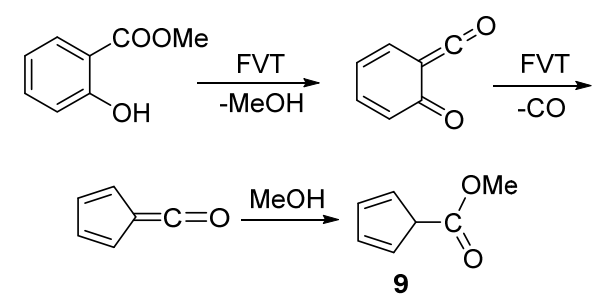

图式 62 2-羟基苯甲酸甲酯合成环戊二烯衍生物

Scheme 6 Synthesis of cyclopentadiene derivatives from 2-hydroxybenzoic acid methyl ester

\subsection{2-环戊烯酮法}

2-环戊烯酮在一定条件下可制备得环戊二烯. 2002 年, Siemeling 等 ${ }^{[48]}$ 利用 2,3,4,5-四甲基-2-环戊烯酮和由 2-溴噻吩与 $\mathrm{BuLi}$ 反应生成的中间体进行亲核加成反应, 再在酸性条件下脱水制得化合物 $\mathbf{1 0}$, 反应式如 Scheme 7 所示.

2014 年, Morris 等 ${ }^{[49]}$ 以 2,3,4,5-四甲基-2-环戊烯酮 为原料, 在 $\mathrm{THF}$ 溶剂中, 与 $\mathrm{RMgBr}$ 反应回流 $3 \mathrm{~h}$, 再经 酸化制得了环戊二烯衍生物. 如 Eq. 5 所示.

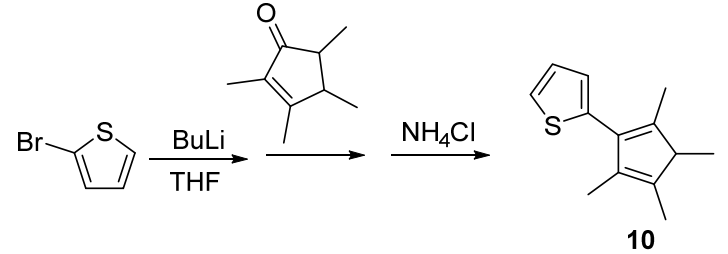

图式 7 1,3,4,5-四甲基-2-噻吩基-1,3-环戊二烯的合成 Scheme 7 Synthesis of 1,2,4,5-tetramethyl-3-thiophenyl-1,3cyclopentadiene

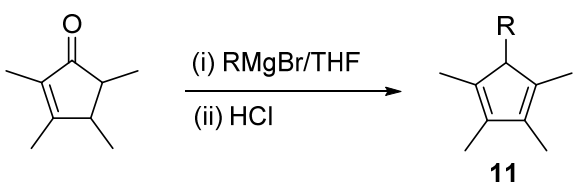

\section{5 联苯甲酰和丙酮反应法}

2014 年, $\mathrm{Pal}$ 等 ${ }^{[50]}$ 将二苯乙二酮与丙酮衍生物在碱 性条件下，乙醇溶液中回流制备环戊二烯酮. 反应式如 Eq. 6 所示.

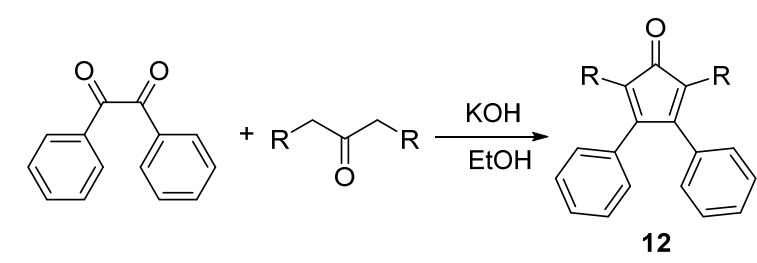

环戍二烯酮作为电子受体单元的可用于 $\mathrm{p}$-型半导 体材料 ${ }^{[7]}$. 此外, 经修饰后的环戊二烯酮聚合物在可见 光波长范围内有较强的吸收带, 在太阳能电池方面有很 好的应用前景，可用于制备的本体异质结器件.

\section{6 其他合成方法}

近年来，非金属催化剂条件下合成环戊二烯衍生物 的方法不断被改进，新的合成路线也不断被开发. 例如， 2013 年, Geng 等 ${ }^{[11]}$ 用一锅合成法合成了一系列四取代 环戊二烯。这种方法不仅操作简单，而且产率很高. 炔 烃衍生物在 THF 溶剂中与二丁基二茂锆 $\left(\mathrm{Cp}_{2} \mathrm{ZrBu}_{2}\right)$ 反应 生成中间产物 ${ }^{[51]}$, 然后在氯化亚铜 $(\mathrm{CuCl}) 、 1,3$-二甲基3,4,5,6-四氢-2-嘧啶酮(DMPU)和 $\mathrm{CH}_{2} \mathrm{I}_{2}$ 作用下生成化合 物 13 (Scheme 8).

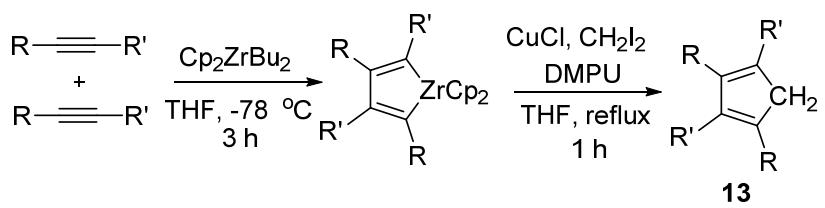

图式 8 炔衍生物合成环戌二烯衍生物

Scheme 8 Synthesis of cyclopentadiene derivatives from alkyne derivatives

Fang 等 ${ }^{[13]}$ 通过方法优化, 利用炔醇与乙烯衍生物 
在有机催化剂(如三氟化硼乙醚)条件下反应制备环戊二 烯衍生物(Eq. 7).

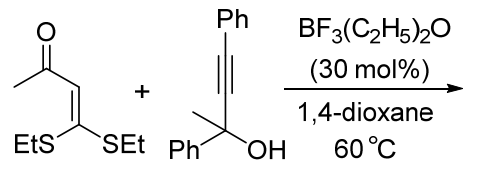

2013 年, Mehdi 等 ${ }^{[2]}$ 将香豆素衍生物和等物质的量 的丁炔二酸二甲酯、叔丁基乙氰反应得到化合物 15 (Eq. $8)$.

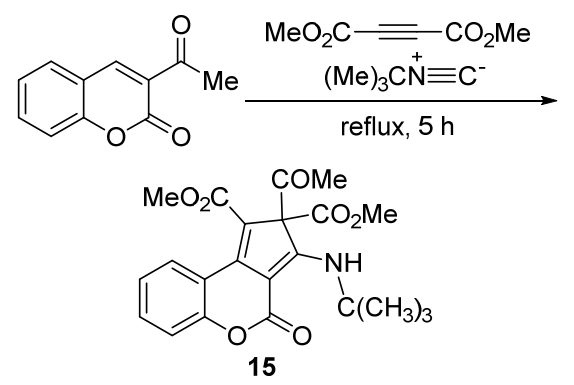

\section{3 环戊二烯及衍生物在合成领域的应用}

环戍二烯及其衍生物在工业生产及制备精细化工 产品和有机合成原料等方面中具有重要的应用价值, 可 用于合成树脂、橡胶、农药、香料、金刚烷、阻燃剂、 降冰片烯类衍生物、环氧树脂固化剂和共聚物等 ${ }^{[53]}$. 这 里主要介绍一些典型环成二烯衍生物在合成领域中的 研究进展, 如金属配位、取代和氧化反应.

\section{1 合成金属有机化合物}

过渡金属配合物是一类在温和条件下具有良好活 性的催化剂, 这种催化剂不仅选择性能高, 并且可回收 重复利用. 金属茂化合物已被广泛应用于催化、太阳能、 储氢、癌症治疗和纳米磁学等领域 ${ }^{[4]}$. 环戊二烯亚甲基 上的氢原子具有很高的反应活性, 游离态环戊二烯呈负 离子状态, 可以与金属络合生成络合物. 1956 年, Piper 等 ${ }^{[55]}$ 利用简单的热反应将 $\mathrm{Fe}(\mathrm{CO})_{5}$ 和环伐二烯反应生成 $\mathrm{Cp}_{2} \mathrm{Fe}_{2}(\mathrm{CO})_{4}$ (Scheme 9).

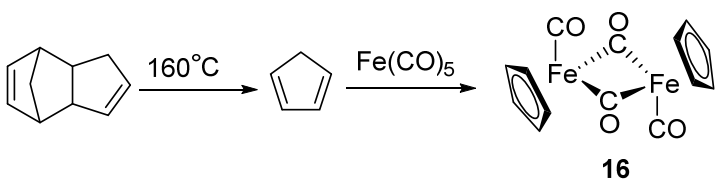

图式 9 环戍二烯与五羰基铁的反应

Scheme 9 Reaction of cyclopentadiene with iron pentacarbonyl

由于原料廉价, 制备简易等优点, $\mathrm{Cp}_{2} \mathrm{Fe}_{2}(\mathrm{CO})_{4}$ 成为 重要的有机铁衍生物试剂. 由于铁是生物体生命循环的
基本元素之一, 因此此类有机铁化合物具有更好的生物 相容性. 环戊二烯基羰基配体化合物由于其独特的性质 在金属配体化合物中有广泛的应用. 2015 年, Jiang 等 ${ }^{[56]}$ 将 $\mathrm{Cp}_{2} \mathrm{Fe}_{2}(\mathrm{CO})_{4}$ 与卤化氢进一步反应生成了具有光敏感 度的 $\left\{\mathrm{Fe}(\text { cis-CO })_{2}\right\}$ 化合物(Eq. 9), 并指出其光敏感性与 配体有关.

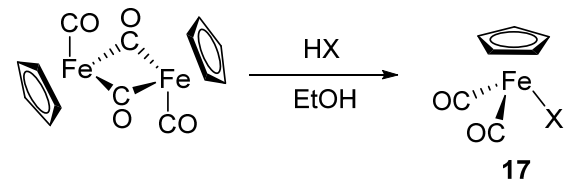

部分有机钉化合物有良好的生物活性，在体内具有 很好的抗转移性能, 可作为抗肿瘤药物应用于医学方 面 ${ }^{[8]}$. 不同的取代基对其生物性能会产生影响 ${ }^{[57]} .2011$ 年, Micallef 等 ${ }^{[58]}$ 对合成的钉金属夹心配位化合物的官 能团进行了修饰研究(Scheme 10).

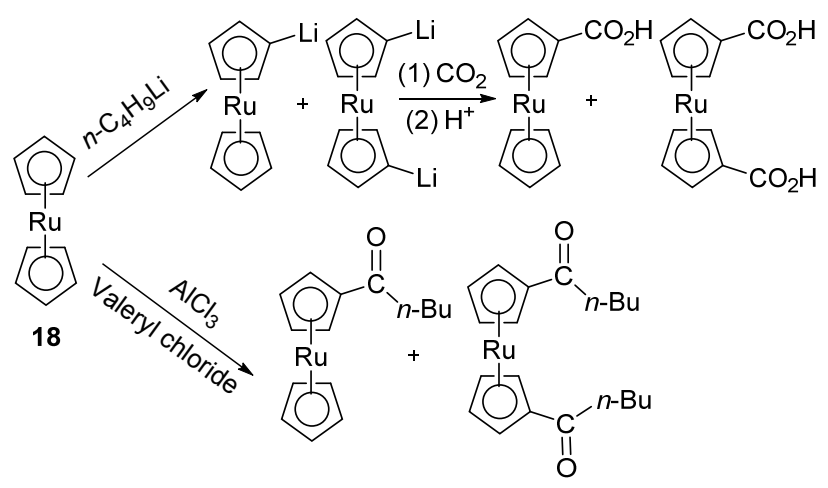

图式 10 二茂钉衍生物的合成

Scheme 10 Synthesis of ruthenocene derivatives

在众多方法中 ${ }^{[59,60]}$, 通过直接催化 $\mathrm{C}-\mathrm{H}$ 键活化可 合成不同的茂金属配合物，此方法是合成不对称金属茂 化合物有效的方法 ${ }^{[61]} .1997$ 年, Siegel 等 ${ }^{[62]}$ 在铜催化条 件下将卡宾插入到二茂铁的环戊二烯的 $\mathrm{C}-\mathrm{H}$ 键中. 2013 年, Gao 等 ${ }^{[63]}$ 发现了靶催化茂金属环戊二烯环上

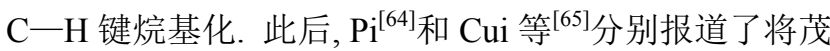
金属化合物中环戊二烯环上的 $\mathrm{C}-\mathrm{H}$ 键烯基和酰化.

\section{2 合成烯烃衍生物}

环戊烯可作为原材料和中间材料用于制备具有特 殊功能的橡胶、环戊酩、环戊醇、溴代环戊烷和氯代环 戊烷等在化学工业领域具有重要应用价值的化工产品. 环戊二烯能被氢气氢化为环戊烯, 但易被进一步还原为 环戊烷, 其中催化剂在此过程中起主要作用. 因此, 探 索和开发新型催化剂高效率地合成环戊烯对科研工作 者来说是一个很大的挑战.

Plate 等 ${ }^{[66]}$ 选用雷尼镍作为环戊二烯氢化还原催化 剂, 但催化剂选择性低、产率低和副产品多. 1997 年, 
Athawale ${ }^{[67]}$ 采用气相环戊二烯在 $\mathrm{Pd} 、 \mathrm{Pt}$ 和 $\mathrm{Ru}$ 等贵金属 催化条件下合成环戍烯, 此方法虽然高效, 但存在催化 剂成本高、制备复杂和寿命短等问题. 近年来, 纳米金 属催化剂尤其是双金属片纳米催化剂由于其高催化性 能、高稳定性和易于回收等特点, 备受外界关注. 纳米 金属催化剂已被用于芳烃和烯烃的氢化还原 ${ }^{[68]}$, 但鲜 有报道用于环戊二烯的氢化还原. 2015 年, Feng 等 ${ }^{[2]}$ 合 成了 $\mathrm{Al}_{2} \mathrm{O}_{3} @ \mathrm{Pd}-\mathrm{Ni}$ 双金属纳米催化剂, 并以其作为非均 相催化剂应用于环戊二烯氢化合成环戊烯(Eq. 10). 此 方法不仅反应条件温和, 还具有较高的转化率和选择 性，因此在商业化生产中具有潜在的应用价值.

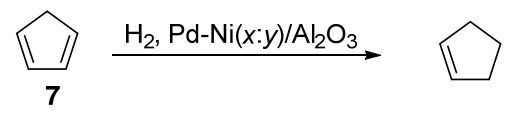

环戍二烯可以直接被单线态的氧气氧化发生开环 反应合成烯酮化合物. 由于环戊二烯双键的第一解离能 小于 $\mathrm{C}-\mathrm{C}$ 单键的解离能, 所以环戊二烯分子的开环反 应趋向从双键加成开始. Hoops 等 ${ }^{[3]}$ 利用单线态的氧气 先与环戊二烯发生 Diels-Alder 反应生成极不稳定的环 状过氧化中间产物, 此化合物在温度高于 $0{ }^{\circ} \mathrm{C}$ 时即发 生 Claisen 重排转化成烯酮化合物(Scheme 11).

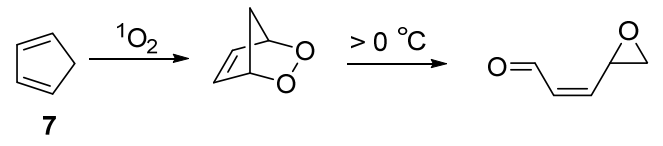

图式 11 1,3-环戊二烯与单线态氧气的反应

Scheme 11 Reaction of 1,3-cyclopentadiene with singlet oxygen

环戍二烯的 Diels-Alder 反应的高反应活性使其在 有机合成 ${ }^{[69]}$ 、生物分子固定化、热敏聚合物和功能性材 料等方面具有广泛的应用 ${ }^{[70,71]}$. 2015 年, Levandowski 等 ${ }^{[4]}$ 利用 Diels-Alder 反应选择性合成了桥式结构化合物 (Eq. 11).

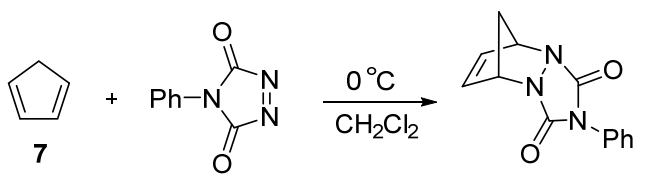

环戊二烯不仅能作为 $4 \pi$ 化合物, 得到桥接的双环 化合物, 也可以为亲二烯体提供逆电子需求进行杂 Diels-Alder (HAD) 反应 ${ }^{[72]}$. Desimoni 等 ${ }^{[5]}$ 利用环戊二烯 的这种特性得到两种不同结构的产物(Scheme 12).

HDA 反应立体选择性较差, 在有机合成领域的应 用价值不大. 2015 年, Gotoh 等 ${ }^{[73]}$ 合成了具有优良选择 性能的有机催化剂, 能有效地调控这两种反应的类型.

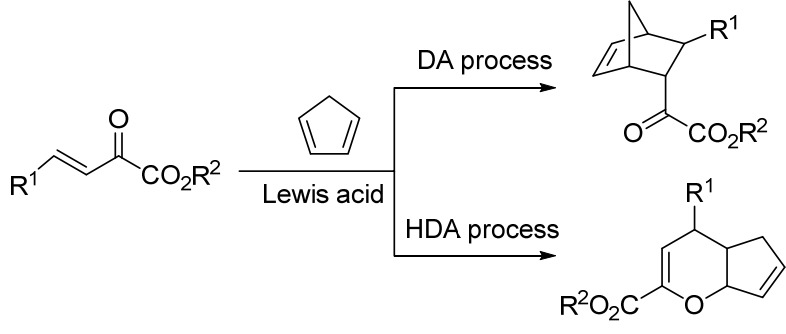

图式 12 1,3-环戊二烯与烯烃衍生物的反应

Scheme 12 Reaction of 1,3-cyclopentadiene with olefin derivatives

\section{5 合成芳香烃}

叔丁基环戊二烯可通过键重排得到甲苯. 中间体双 环化合物的环丙基结构是由内部的一个双键断裂形成, 然后经过 $\mathrm{C}-\mathrm{C}$ 键断裂重组最终形成甲苯 ${ }^{[6]}$, 如 Scheme 13 所示.
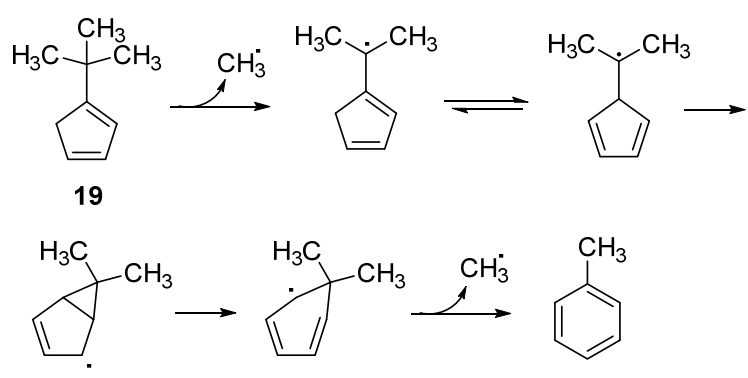

图式 13 5-叔丁基-1,3-环戊二烯合成甲苯

Scheme 13 Synthesis of toluene from 5- tert-butyl-1,3-cyclopentadiene

\section{6 合成聚合物}

由于共轭双键的作用，环戊二烯亚甲基上的氢非常 活泼，当与活泼金属和强碱作用时可得到具有一定稳定 性的环戊二烯负离子. 环戊二烯负离子具有独特的结构 和较好的反应活性, 不仅能合成多种环戊二烯衍生物和 金属茂配合物，也可制备具有高比表面积和良好物理化 学稳定性的微孔有机聚合物(MOPS).

2012 年, Liu 等 ${ }^{[74]}$ 指出过度金属催化的偶联反应往 往面临投资高，环境污染大，纯化困难等问题，这是大 分子偶联反应的重大难题. 2013 年, Kim 等 ${ }^{[9]}$ 以 1,3,5-三 溴甲基-2,4,6-三甲基苯为原料在 $\mathrm{THF}$ 溶剂中, $0{ }^{\circ} \mathrm{C}$ 条件 下与环戊二烯钠反应制备了具有微孔结构的聚合物(Eq. 12). 与以往的微孔聚合物相比, 此交联聚合物具有较 大的比表面积和较好的性能. 此反应具有无需金属催 化，操作简单和反应条件温和等优点.

\section{7 合成官能团衍生物}

2014 年, Jorner 等 ${ }^{[75]}$ 利用环戊二烯在氢氧化钠作用 下生成的环戍二烯钠盐与卤代化合物反应合成环戊二 
<smiles>Cc1c(CBr)c(C)c(CBr)c(C)c1CBr</smiles>

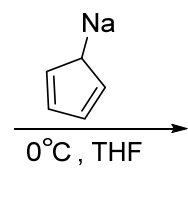<smiles>Cc1c(C)c(C)c(C2=CCC=C2)c(C)c1C</smiles><smiles>Cc1c(C)c(C)c(CC2=CC3CC2CC3Cc2c(C)c(C)c(C)c(CC3=CC4CC3C3C=CCC43)c2C)c(C)c1C</smiles>

烯衍生物(Scheme 14).

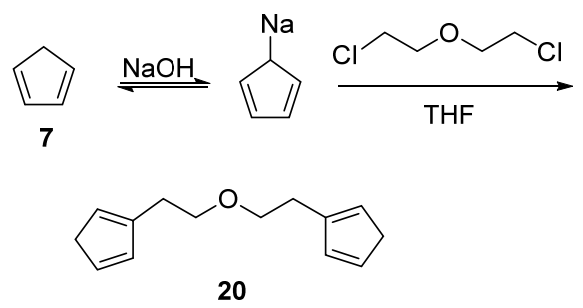

图式 14 1,3-环戊二烯与 2,2'-二氯二乙基醚的反应 Scheme 14 Reaction of 1,3-cyclopentadiene with 2,2'-dichlorodiethyl ether

环戊二烯可与醛或酮反应得到富烯, 这种共轭结构 增强了环键的稳定性, 在合成茂金属络合物方面具有重 要的应用. 2014 年, Sha 等 ${ }^{[76]}$ 利用环戊二烯和环已酮反 应合成了富烯衍生物(Eq. 13). 作为富烯化合物的衍生 物之一, 五富烯化合物具有很强的电子接受性能 ${ }^{[77]}$, 并 可通过改变电子分布适应其芳香性的不同性质 ${ }^{[78]}$, 因 此在合成领域具有非常重要的应用.

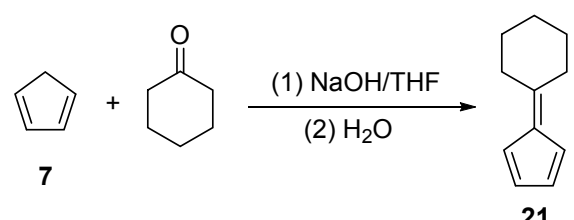

\section{9 合成有机中间体}

烯丙位有一个供电子基团取代的环戊二烯在(超)强 酸作用下烯丙位上的氢以负离子形式脱去, 使烯丙位碳 原子失去电子形成环戊二烯碳正离子 ${ }^{[79]}$. 环戊二烯碳 正离子易与活拨金属反应生成较稳定的环戊二烯金属 盐, 也能与烷烃或过渡金属盐反应得到烷基取代的环戊 二烯或有机金属茂化合物. 五甲基环戊二烯可经过质子 化得到较稳定的阳离子化合物 (Eq. 14) ${ }^{[80]}$. 而该类碳正 离子为重要的有机合成媒介, 例如五甲基环戊烯基阳离 子被证明是 MTO 的重要中间体 ${ }^{[81]}$.

过渡金属, 如高氯酸银, 作为氧化剂可以氧化多苯 基取代环戊二烯生成带正电的氧环化合物吡喃盐. 吡喃

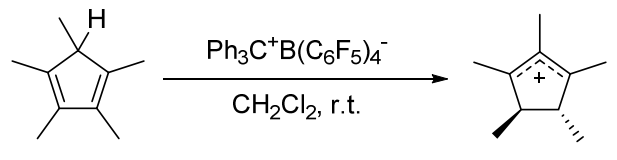

22

盐是一种重要的合成中间体, 在有机合成方面已得到广 泛的研究. 吡喃盐具有独特的光物理和电化学性能, 在 激光染料、 $\mathrm{Q}$ 开关材料、发光材料以及电照相体系等领 域有着广阔的应用前景 ${ }^{[82,83]}$.

2004 年, 我们课题组 ${ }^{[19]}$ 首次用多芳基环戊二烯在 高氯酸银催化条件下，与 $\mathrm{O}_{2}$ 反应一步合成了具有 $\alpha$-活 性的吡喃盐. 2005 年, 我们发现 ${ }^{[20]}$ 在酸性条件下环戊二 烯也可与氧气反应一步合成吡喃盐. 2013 年, 我们 ${ }^{[21]}$ 通 过合成多种官能团取代的多芳基环戊二烯得到了发光 波长在 $500 \sim 700 \mathrm{~nm}$ 范围内的多色彩吡喃盐化合物 (Eq. 15).
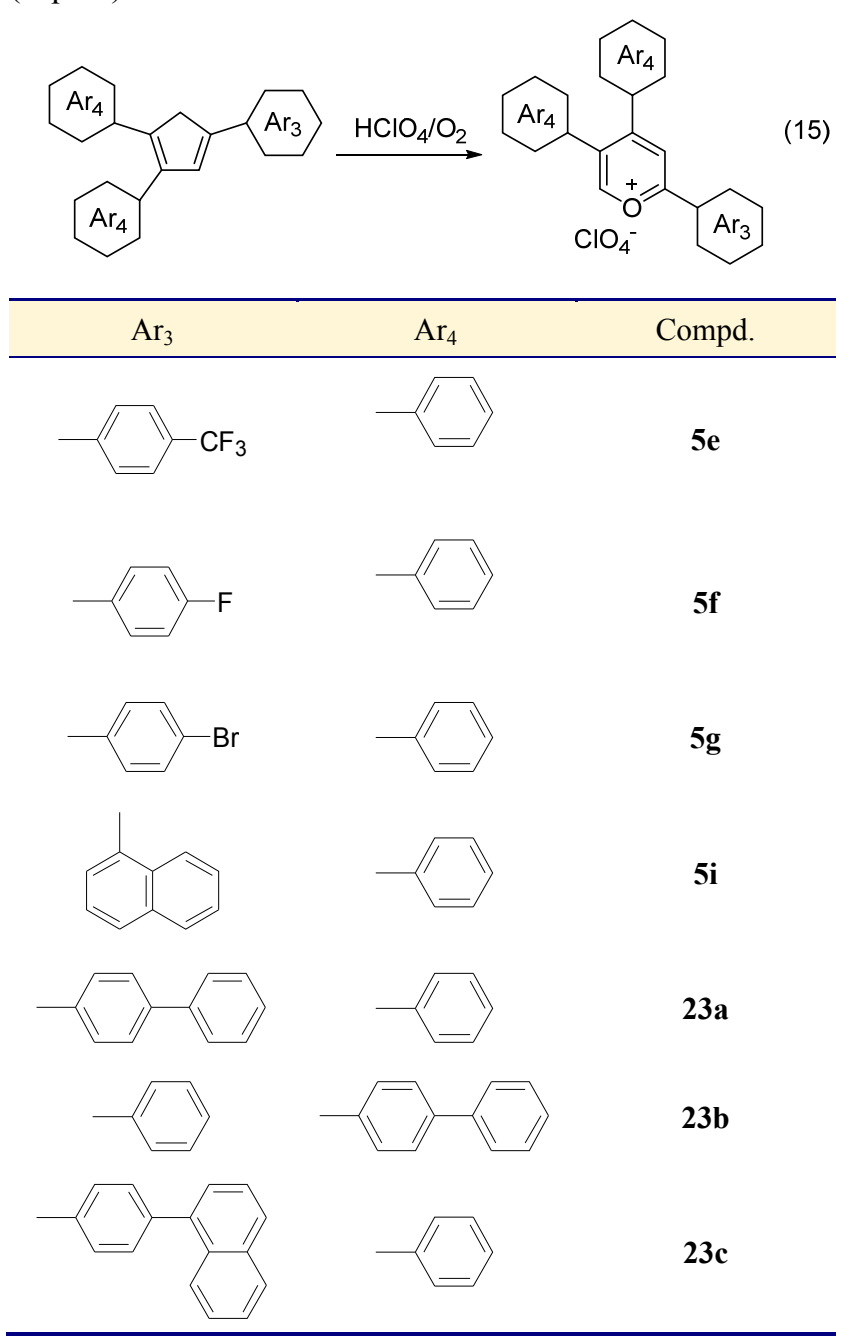

与传统利用过渡金属作氧化剂的合成方法相比，这 种利用高氯酸一步反应合成吡喃盐的方法克服了催化 剂价格昂贵、易失活、污染环境，只能在特定溶剂中反 应和只能合成某些吡喃盐化合物等缺点. 这种方法还具 
有较好的选择性和产率，因此具有良好的应用前景和较 高的研究价值. 我们发现该类反应中间体通过经优化的 $\mathrm{Mg} / \mathrm{CH}_{3} \mathrm{CN}$ 还原体系可发生二聚反应合成多烯酩化合 物, 开辟了合成共轭多烯酮化合物的新途径 ${ }^{[20,24]}$.

2013 年, 我们 ${ }^{[22]}$ 利用 $\mathrm{FeCl}_{3} \cdot 6 \mathrm{H}_{2} \mathrm{O}$ 催化多芳基环戊 二烯在甲苯和二氯甲烷混合溶剂中反应, 得到一系列非 $\alpha$-活性吡喃盐化合物 (Eq. 16). 此方法不仅反应条件温 和, 步骤简单, 还具有选择性强、产率高等优点. 此外, 合成的四氯化铁吡喃盐具有很好的发光性能和水溶性.
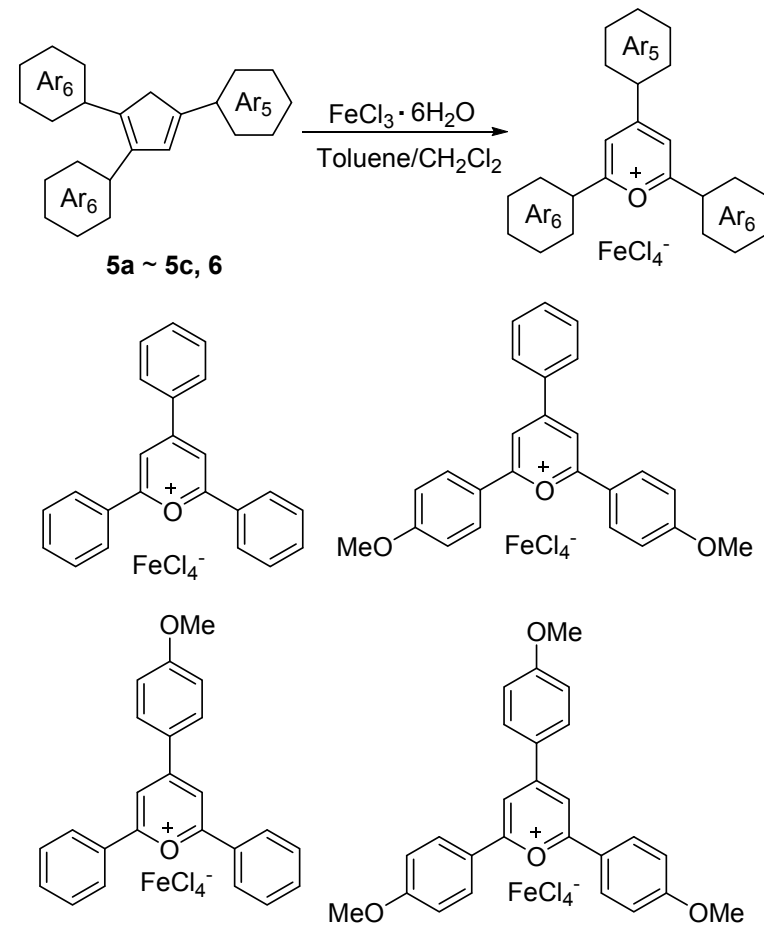

\section{4 结论和展望}

综上所述，环戈二烯及其衍生物由于其较高的反应 活性及性能, 在化学反应研究和工业化工生产中都具有 广泛应用. 由于环戊二烯在常温下易于聚合不能以单分 子态存在, 合成能在常温下稳定存在的环戊二烯衍生物 具有重要的意义. 随着环境的恶化与绿色无污染生产的 倡导, 无金属催化剂尤其光化学诱导合成环戊二烯及其 衍生物的方法也将会引起越来多的关注, 并具有深远的 意义. 此外, 多芳基取代环戊二烯作为一类有机小分子 发光材料, 具有聚集诱导发光和纳米聚集态多色苂光发 射性能，在有机发光领域的应用吸引了人们极大的关 注. 通过分子设计, 可以将多芳基环戊二烯衍生物应用 于光电器件中, 此类材料具有很好的潜在应用价值. 随 着合成化学的发展，性能更丰富的环戊二烯衍生物将不 断涌现，在有机合成中间体、有机发光材料、苂光传感、 光敏材料、高分子材料、催化、航空煤油及燃料添加剂
等领域会有更为广泛的应用.

\section{References}

[1] Tjahjono, A. M.; Feng, G.; Hermanto, M. W.; Cechao, F.; Garland, M. RSC Adv. 2014, 4, 22194.

[2] Feng, Y.-S.; Hao, J.; Liu, W.-W.; Yao, Y.-J.; Cheng, Y.; Xu, H.-J. Chin. Chem. Lett. 2015, 26, 709.

[3] Hoops, M. D.; Ault, B. S. J. Am. Chem. Soc. 2009, 131, 2853.

[4] Levandowski, B. J.; Houk, K. N. J. Org. Chem. 2015, 80, 3530.

[5] Desimoni, G.; Faita, G.; Toscanini, M.; Boiocchi, M. Chem. Eur. J. 2007, 13, 9478.

[6] McGivern, W. S.; Manion, J. A.; Tsang, W. J. Phys. Chem. A 2006, $110,12822$.

[7] Yang, C.; Cho, S.; Chiechi, R. C.; Walker, W.; Coates, N. E.; Moses, D.; Heeger, A. J.; Wudl, F. J. Am. Chem. Soc. 2008, 130, 16524.

[8] Suess-Fink, G. Dalton Trans. 2010, 39, 1673.

[9] Kim, H.; Choi, T.; Cha, M. C.; Chang, J. Y. J. Polym. Sci., Part A: Polym. Chem. 2013, 51, 3646.

[10] Rettenmeier, E.; Schuster, A. M.; Rudolph, M.; Rominger, F.; Gade, C. A.; Hashmi, A. S. K. Angew. Chem. Int. Ed. 2013, 52, 5880.

[11] Geng, W. Z.; Wang, C.; Guang, J.; Hao, W.; Zhang, W.-X.; Xi, Z. F. Chem. Eur. J. 2013, 19, 8657.

[12] Wirtanen, T.; Muuronen, M.; Melchionna, M.; Patzschke, M.; Helaja, J. J. Org. Chem. 2014, 79, 10269.

[13] Fang, Z. X.; Liu, J. Q.; Liu, Q.; Bi, X. H. Angew. Chem. Int. Ed. 2014, 53, 7209.

[14] Yang, L. J.; Ye, J. W.; Xu, L. F.; Yang, X. Y.; Gong, W. T.; Lin, Y.; Ning, G. L. RSC $A d v . \mathbf{2 0 1 2}, 2,11529$.

[15] Zhang, X. D.; Ye, J. W.; Xu, L. F.; Yang, L. J.; Deng, D.; Ning, G. L. J. Lumin. 2013, 139, 28.

[16] Ye, J. W.; Deng, D.; Gao, Y.; Wang, X. X.; Yang, L. J.; Lin, Y.; Ning, G. L. Spectrochim. Acta, Part A 2015, 134, 22.

[17] Ye, J.W.; Gao, Y.; He, L.; Tan, T. T.; Chen, W.; Liu, Y.; Wang, Y.; Ning, G. L. Dyes Pigm. 2016, 124, 145.

[18] Ye, J. W.; Xu, L. F.; Gao, Y.; Wang, H.; Ding, Y. Z.; Deng, D.; Gong, W. T.; Ning, G. L. Synth. Met. 2013, 175, 170.

[19] Ning, G. L.; Li, X. C.; Munakata, M.; Gong, W. T.; Maekawa, M.; Kamikawa, T. J. Org. Chem. 2004, 69, 1432.

[20] Gong, W. T.; Ning, G. L.; Li, X. C.; Wang, L.; Lin, Y. J. Org. Chem. 2005, 70, 5768.

[21] Yang, L. J.; Ye, J. W.; Gao, Y.; Deng,D.; Gong, W. T.; Li, Y.; Ning, G. L.; Tetrahedron Lett. 2013, 54, 2967.

[22] Ye, J. W.; Zhang, X. D.; Deng, D.; Ning, G. L.; Liu, T. Q.; Zhuang, M. L.; Yang, L. J.; Gong, W. T.; Lin, Y. RSC Adv. 2013, 3, 8232.

[23] Zhang, X. D.; Ye, J. W.; Wang, S. N.; Gong, W. T.; Lin, Y.; Ning, G. L. Org. Lett. 2011, 13, 3608 .

[24] Yang, L. J.; Ye, J. W.; Gao, Y.; Deng, D.; Lin, Y.; Ning, G. L. Eur. J. Org. Chem. 2014, 515

[25] Gong, W. T.; Na, D.; Mehdi, H.; Ye, J. W.; Ning, G. L. J. Organomet. Chem. 2014, $772 \sim 773,314$.

[26] Ye, J. W.; Wang, X. X.; Gao, Y.; Yang, L. J.; Lin, Y.; Ning, G. L. Chin. J. Org. Chem. 2015, 35, 373 (in Chinese) (叶俊伟, 王潇潇, 高远, 杨立健, 林源, 宁桂玲, 有机化学, 2015, 35, 373.)

[27] Li, G.; Gong, W.-T.; Ye, J.-W.; Lin, Y.; Ning, G.-L. Synth. Commun. 2012, $42,480$.

[28] Li, G.; Gong, W. T.; Ye, J. W.; Lin, Y.; Ning, G. L. Chin. J. Org. Chem. 2011, 31, 216 (in Chinese). (李刚, 贡卫涛, 叶俊伟, 林源, 宁桂玲, 有机化学, 2011, 31, 216.) 
[29] Peng, S. Y.; Gao, T.; Sun, S. F.; Peng, Y. H.; Wu, M. H.; Guo, H. B.; Wang, J. Adv. Synth. Catal. 2014, 356, 319.

[30] Dömling, A. Chem. Rev. 2006, 106, 17.

[31] Chen, L.; Mahmoud, S. M.; Yin, X. D.; Lalancette, R. A.; Pietrangelo A. J. Am. Chem. Soc. 2013, 15, 5970.

[32] Loh, C. C. J.; Enders, D. Chem. Eur. J. 2012, 18, 10212.

[33] Becker, L.; Burlakov, V. V.; Arndt, P.; Spannenberg, A.; Baumann, W.; Jiao, H. J.; Rosenthal, U. Chem. Eur. J. 2013, 19, 4230.

[34] Kryukov, S. I.; Dzyuba, I. V.; Smirnov, V. A. Neftekhimiya 1991, $31,386$.

[35] Braga, A. A. C.; Morgon, N. H.; Ujaque, G.; Maseras, F. J. Am. Chem. Soc. 2005,127, 9298.

[36] Du, Y. M.; Li, C. Y.; Zhang J. W.; Wang, W.; Kang, J. P.; Lv, J. Chem. J. Chin. Univ. 2014, 35, 755 (in Chinese).

(杜咏梅, 李春迎, 张建伟, 王 伟, 六建平, 吕剑, 高等学校化 学学报, 2014, 35, 755.)

[37] Li, L.; Cai, Z.; Shen, B.; Xin, Z.; Ling, H. Chem. Eng. Technol. 2011, 34, 1468.

[38] Khambata, B. S.; Wassermann, A. J. Chem. Soc. 1939, 375.

[39] Krupka, J. Pet. Coal. 2010, 52, 290.

[40] Goswami, T.; Das, D. K.; Goswami, D. Chem. Phys. Lett. 2013, $558,1$.

[41] Herndon, W. C.; Grayson, C. R.; Manion, J. M. J. Org. Chem. 1967, $32,526$.

[42] Wang, W.; Chen, J.-G.; Song, L.-P.; Liu, Z.-T.; Liu, Z.-W.; Lu, J.; Xiao, J. L.; Hao, Z. P. Energy Fuels 2013, 27, 6339.

[43] Wang, W. T.; Cong, Y.; Chen, S.; Sun, C. X.; Wang, X. D.; Zhang, T. Top. Catal. 2015, 58, 350.

[44] Mendez, I. D.; Klimova, E.; Klimova, T.; Hernández, O. S.; Martí nez, G. M. J. Organomet. Chem. 2003, 681, 115.

[45] Kollenz, G.; Ebner, S. Sci. Synth. 2006, 23, 271.

[46] Scheer, A. M.; Mukarakate, C.; Robichaud, D. J.; Nimlos, M. R.; Carstensen, H.-H.; Barney Ellison, G. J. Chem. Phys. 2012, 136, 044309.

[47] Koch, R.; Blanch, R. J.; Wentrup, C. J. Org. Chem. 2014, 79, 6978.

[48] Siemeling, U.; Neumann, B.; Stammler, H.-G.; Salmon, A. Z. Anorg. Allg. Chem. 2002, 628, 2315.

[49] Morris, D. M.; McGeagh, M.; De Peña, D.; Merola, J. S. Polyhedron 2014, 84, 120.

[50] Pal, R.; Mukherjee, S.; Chandrasekhar, S.; Guru Row, T. N. J. Phys. Chem. A 2014, 118, 3479.

[51] Kaleta, K.; Strehler, F.; Hildebrandt, A.; Beweries, T.; Arndt, P.; Rüffer, T.; Spannenberg, A.; Lang, H.; Rosenthal, U. Chem. Eur. J. 2012, 18, 12672.

[52] Mehdi, G.; Ali-Tabatabaei, G.; Maciej, K. J. Org. Chem. 2013, 78, 2611.

[53] Wang, Y. X.; Duan, X. M.; Wang, Q.; Li, Y. S.; Liu, J. Y. Acta Chim. Sinica 2011, 69(18), 2085 (in Chinese). (王永霞, 段雪梅, 王钦, 李悦生, 刘靖尧, 化学学报, 2011, 69(18), 2085.)
[54] Wu, X. J.; Zeng, X. C. J. Am. Chem. Soc. 2009, 131, 14246.

[55] Piper, T. S.; Wilkinson, G. J. Inorg. Nucl. 1956, 3(2), 104.

[56] Jiang, X. J.; Chen, L. M.; Wang, X.; Long, L.; Xiao, Z. Y.; Liu, X. M. Chem. Eur. J. 2015, 21, 13065.

[57] Loughrey, B. T.; Williams, M. L.; Carruthers, T. J.; Parsons, P. G.; Healy, P. C. Aust. J. Chem. 2010, 63, 245.

[58] Micallef, L. S.; Loughrey, B. T.; Healy, P. C.; Parsons, P. G.; Williams, M. L. Organometallics 2011, 30, 1395.

[59] Schaarschmidt, D.; Lang, H. Organometallics 2013, 32, 5668.

[60] Ogasawara, M.; Watanabe, S.; Nakajima, K.; Takahashi, T. J. Am. Chem. Soc. 2010, 132, 2136.

[61] Zheng, C.; You, S.-L. RSC Adv. 2014, 4, 6173.

[62] Siegel, S.; Schmalz, H.-G. Angew. Chem., Int. Ed. 1997, 36, 2456.

[63] Gao, D.-W.; Shi, Y.-C.; Gu, Q.; Zhao, Z.-L.; You, S.-L. J. Am. Chem. Soc. 2013, 135, 86.

[64] Pi, C.; Li, Y.; Cui, X. L.; Zhang, H.; Han, Y. B.; Wu, Y. J. Chem. Sci. 2013, 4, 2675.

[65] Pi, C.; Cui, X. L.; Liu, X. Y.; Guo, M. X.; Zhang, H. Y.; Wu, Y. J. Org. Lett. 2014, 16, 5164.

[66] Plate, A. F.; Stanko, V. I. Bull. Acad. Sci. USSR, Div. Chem. Sci. 1956, $5,1173$.

[67] Athawale, V. D.; Rathi, S. C. J. Appl. Polym. Sci. 1997, 66 , 1399.

[68] Fernando, C.-L.; Santiago, G.-Q.; Claudia, A.; Keane, M. A. Appl. Catal. A 2014, 473, 41

[69] Shi, Y.; Wilmot, J. T.; Nordstrøm, L. U.; Tan, D. S.; Gin, D. Y. J. Am. Chem. Soc. 2013, 135,14313.

[70] Samoshin, A. V.; Hawker, C. J.; Read de Alaniz, J. ACS Macro. Lett. 2014, 3, 753.

[71] Bian, S.; Scott, A. M.; Cao, Y.; Liang, Y.; Osuna, S.; Houk, K. N.; Braunschweig, A. B. J. Am. Chem. Soc. 2013, 135, 9240.

[72] Kouznetsov, V. V. Tetrahedron 2009, 65, 2721.

[73] Gotoh, H.; Uchimaru, T.; Hayashi, Y. Chem. Eur. J. 2015, 21, 12337.

[74] Liu, J. H.; Lei, M.; Hu, L. H. Green Chem. 2012, 14, 2534.

[75] Jorner, K.; Emanuelsson, R.; Dahlstrand, C.; Tong, H.; Denisova, A. V.; Ottosson, H. Chem. Eur. J. 2014, 20, 9295.

[76] Sha, L.; Li, L.; Yuan, F. G. Chin. J. Chem. 2014, 32, 1214.

[77] Finke, A. D.; Diederich, F. Chem. Rec. 2015, 15, 19.

[78] Dahlstrand, C.; Rosenberg, M.; Kilså, K.; Ottosson, H. J. Phys. Chem. A 2012, 116, 5008.

[79] Alonso, M.; Herradón, B. Phys. Chem. Chem. Phys. 2010, 12, 1305.

[80] Müller, T. Angew. Chem., Int. Ed. 2002, 41, 2276.

[81] Xu, S. T.; Zheng, A.; Wei, Y. X.; Chen, J. R.; Li, J. Z.; Chu, Y. Y.; Zhang, M. Z.; Wang, Q. Y.; Zhou, Y.; Wang, J. B.; Deng, F.; Liu, Z. M. Angew. Chem., Int. Ed. 2013, 52, 11564.

[82] Nikolov, P.; Metzov, S. J. Photochem. Photobiol. A: Chem. 2000, 135, 13.

[83] Bello, A. M.; Kotra, L. P. Tetrahedron Lett. 2003, 44, 9271. 